\title{
A NEW APPROACH TO DAMAGE DETECTION IN COMPOSITE BODY STRUCTURES USING TIME COMPENSATED HYPERSPECTRAL IMAGE ANALYSIS TECHNIQUE
}

\author{
Mahmoud Zaki Iskandarani \\ Department Computer Science, Faculty of Science and Information Technology, \\ Al-Zaytoonah University of Jordan, Post Code 11191, Amman, Jordan
}

Received 2012-07-12, Revised 2012-10-02; Accepted 2013-03-16

\begin{abstract}
A novel approach to damage detection in composite car body structures using hyperspectral image index analysis algorithm is presented and discussed. The adapted technique allows the monitoring and analysis of a components structure based on correlation between time spaced thermal images. The technique produces several organized tables resulting from image fusion and frame deviation pixel redistribution calculations, which results in computable matrices. The obtained results proved the technique to be capable of classifying damage with ability to model various types of damage under various conditions. There is a need to accurately identify damaged composite components. This will enable the used algorithm to determine if the level of damage or defect in the component is critical according to established database that takes into account mechanical and physical factors. Testing different composite structures using Hyperspectral technique and Pulse Video Thermography (PVT), as hyperspectral uses different bands with different wavelengths to analyze the image results, which enables better recognition and classification. Successful recognition and component classification obtained with characterization of tested composite.
\end{abstract}

Keywords: Hyperspectral, Infrared Thermography, Image Fusion, NDT, Composites, Damage Detection, PVT

\section{INTRODUCTION}

Advanced layered composite materials are in wide use in many applications. Fiber reinforced composites with polymeric matrices (FRP or laminates) and polymeric sandwich materials, with thin laminate faces and foam or impregnated cores, are examples of such structures. The structural design and maintenance of composite structures involving these materials need comprehensive evaluation and characterization of mechanical properties and behavior under different loading conditions, in both undamaged and damaged state.

The marked inhomogeneity and anisotropy of these materials makes them vulnerable to a variety of damages. For this reason, reliable composite structures need adequate NDT/NDE methods along the maintenance activities and knowledge of residual strength/stiffness or service life estimation linked to certain damage patterns. In the end, development of damage tolerant materials may be considered a goal towards further increasing the attractiveness of composite materials in building high tech reliable products.

As composite materials are finding increasing use in more demanding applications, requiring a high degree of accuracy and reliability, considerable effort is being made to define and setup quality control procedures and inspection methods.

Controlling the quality of raw composite materials is carried out to detect the following:

- Excessive void contents or porosity

- Contamination or foreign particle inclusions

- Variation in the degree of resin cure

- Inconsistent fiber volume fraction

- Dimensional inaccuracies 


\section{- $\quad$ Poor fiber-matrix bonding}

\section{- Broken or Damaged fibers}

A composite structure may also be damaged in service due to physical damage such as impact or fatigue creep. The influence of the environment on a structure due to ingress or moisture, exposure to hot and wet conditions for over long periods, contamination from oils or fluids may also cause damage, like surface abrasion and dents, delamination, fiber crack, bonding failure.

Such defects are all potentially detrimental to the mechanical integrity and consequently to the structural performance of a component. The extent to which a defect will affect the performance will depend on the geometry of the structure, the location and orientation of the defect, the type of applied stress field and the working environment.

Confidence in the application of safety critical structural composites in vehicles would be improved if a fast accurate method of assessing manufacturing flaws and service damage in relation to the structural engineering performance was available. A perceived problem with composite structures is that internal damage may seriously weaken a structure yet be undetected due to little surface evidence.

Infrared thermography has been used as an outstanding non-destructive testing method. Its basic principle is exciting an object thermally/mechanically and using infrared camera to monitor changes of the object's surface temperature. The subsurface discontinuities will heat diffusion, thus will affect the thermal distribution of the surface. Various excitation sources can be used, such as cold/hot air, high-powerful lamps and flashes, sonic/ultrasound transducers and others. There are also different heating techniques. Modulated and pulsed heating are among the most popular ones. There are two modes for thermography, one where the specimen is heated from one side, with thermal data is collected from the same side, called reflection mode and the other where the specimen is heated from one side, while thermal data is collected from the opposite side, called transmission mode.

Pulse Video Thermography (PVT) is a versatile NDT inspection method, ready to be used in industrial applications assisted by computer and intelligent software specifically designed for this purpose.

The subjected material reacts rapidly after the initial thermal pulse because the thermal front propagates, by diffusion, under the surface and also because of radiation and convection losses. The presence of a defect reduces the diffusion rate so that when observing the surface temperature, defects appear as areas of different temperatures with respect to surrounding sound areas once the thermal front has reached them. Consequently, deeper defects will be observed later and with a reduced contrast.

Such approach is common in the automotive industry. Other common applications of the active PVT scheme are in quantitative subsurface defect assessment (cracks, delaminations, impact damages, disbondings, moisture), thermophysical property evaluation; in all kind of industries (Myriounis et al., 2011; Lahiria et al., 2012; Naderi et al., 2012; Wu et al., 2011; Cheng and Tian, 2012; Kutin et al., 2011).

In Hyperspectral image analysis approach, image fusion which is the process of combining relevant information from two or more images into a single image takes place. The resulting image will be more informative than any of the input images.

The image fusion techniques allow the integration of different information sources. The fused image can have complementary spatial and spectral resolution characteristics. Such techniques are usually used in satellite imaging; where two types of images are available. The panchromatic image acquired by satellites is transmitted with the maximum resolution available and the multispectral data are transmitted with coarser resolution. This will usually be two or four times lower. At the receiving end, the panchromatic image is merged with the multispectral data to convey more information (Baranowski et al., 2012; Meer et al., 2012; Yanga et al., 2012; Liu et al., 2011; Picon et al., 2012; Zhao et al., 2011).

In almost all application areas, the basic goal of hyperspectral image analysis is to classify or discriminate objects. Common problems in the area of hyperspectral analysis involving data relevancy include optimal selections of wavelength, number of bands and spatial and spectral resolution.

Despite the fact that hyperspectral image analysis is used to perform index analysis in hyperspectral and multispectral satellite imagery, it is assumed that it can be used for image comparison of similar or processed images, of completely different origin.

In this study the hyperspectral approach is adopted to detect and analyze damage in composite car structures such as a wishbone. The adaption of the technique is to assume the high resolution panachromatic image (PAN) to be the reference image while the image of the damaged component to be the low resolution multispectral image (MS). The success of applying the technique depends on time compensation of sampled images and propagated heat waves. Also, this study will prove the validity and actual advantage of using 
Hyperspectral image index analysis on normal nonsatellite images specifically on PVT images.

\section{MATERIALS AND METHODS}

In this study an algorithm designed to compute four image indices RMSE, RASE, Bias and Deviation. The purpose of the used program is to provide such indices results in a matrix format for further analysis.

The used approach is as follows:

- Computation of relevant image indices

- Formation of individual matrices

- Formation of characteristic Matrix

\section{RESULTS}

Figure 2-6 show wishbone thermal images from a damaged side obtained using the setup in Fig. 1, while Fig. 7-9 present thermal images for the wishbone undamaged side.
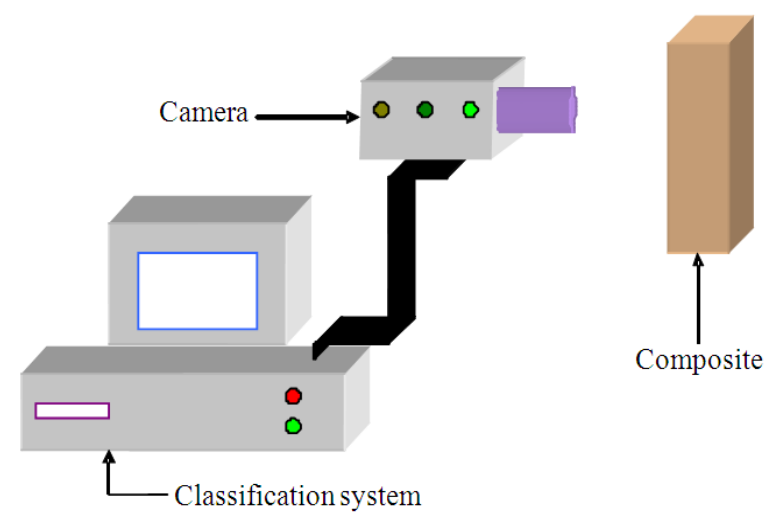

Fig. 1. Experimental setup

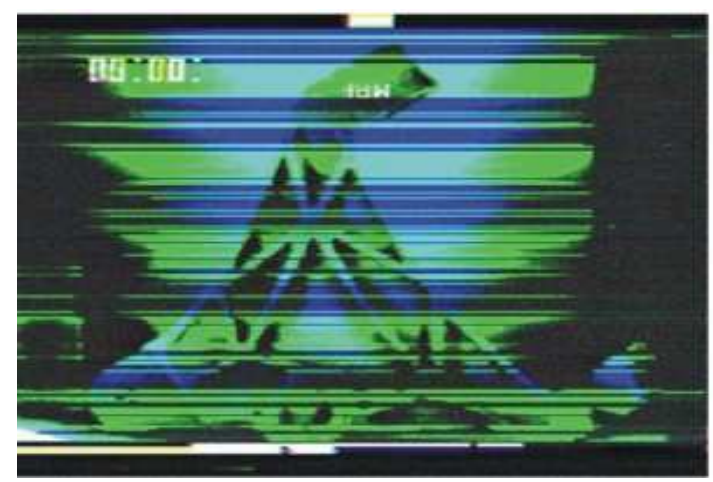

Fig. 2. Damaged Wishbone thermal image at $0 \mathrm{sec}$

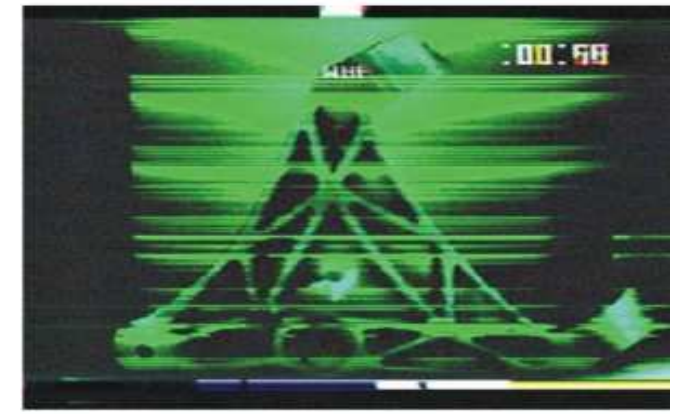

Fig. 3. Damaged Wishbone thermal image at $0.59 \mathrm{sec}$

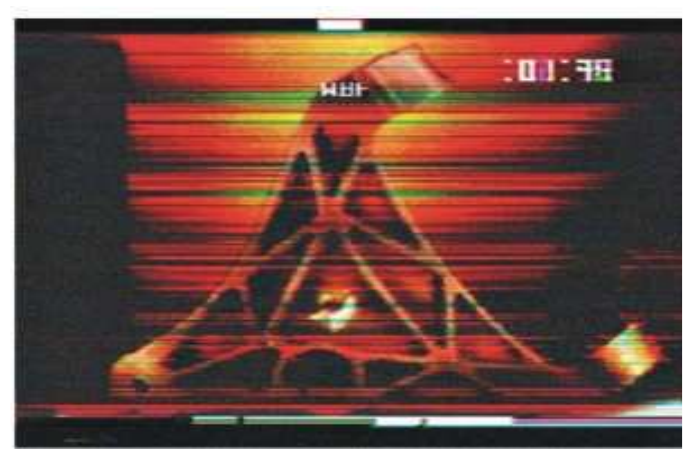

Fig. 4. Damaged Wishbone thermal image at $1.39 \mathrm{sec}$

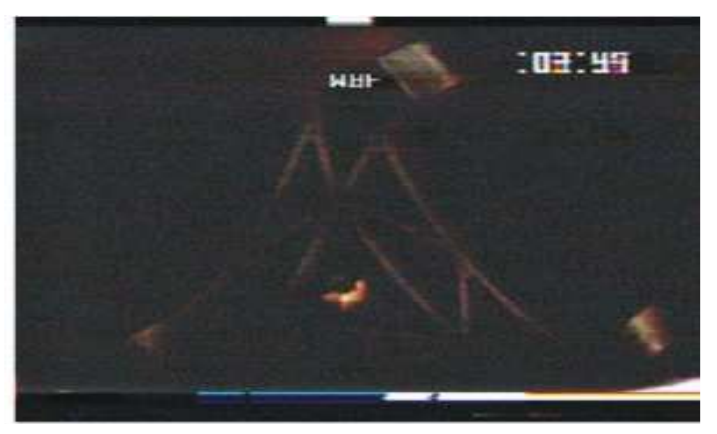

Fig. 5. Damaged Wishbone thermal image at $3.49 \mathrm{sec}$

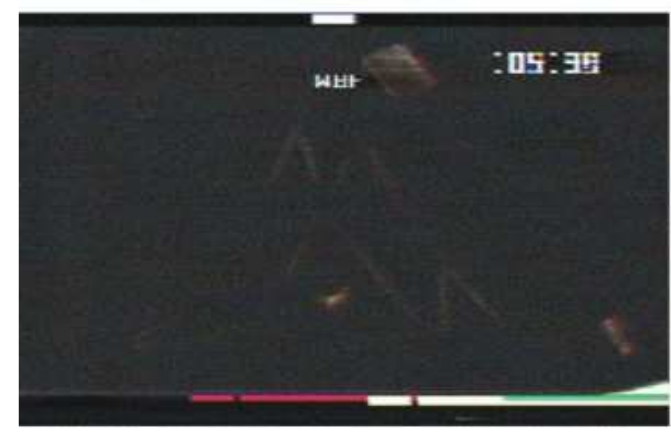

Fig. 6. Damaged Wishbone thermal image at $5.39 \mathrm{sec}$ 
Mahmoud Zaki Iskandarani / American Journal of Applied Sciences, 10 (2): 123-130, 2013

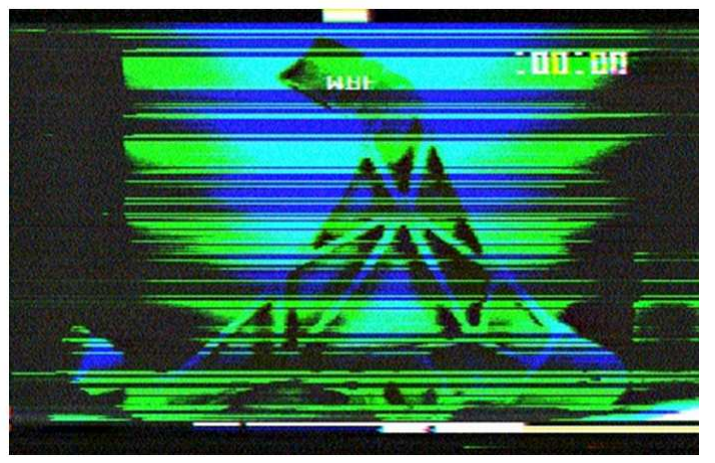

Fig. 7. Undamaged Wishbone thermal image at $0 \mathrm{sec}$

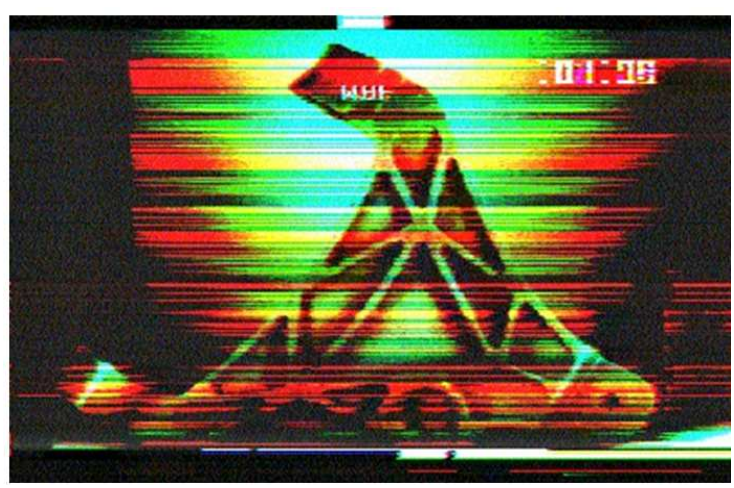

Fig. 8. Undamaged Wishbone thermal image at $1.26 \mathrm{sec}$

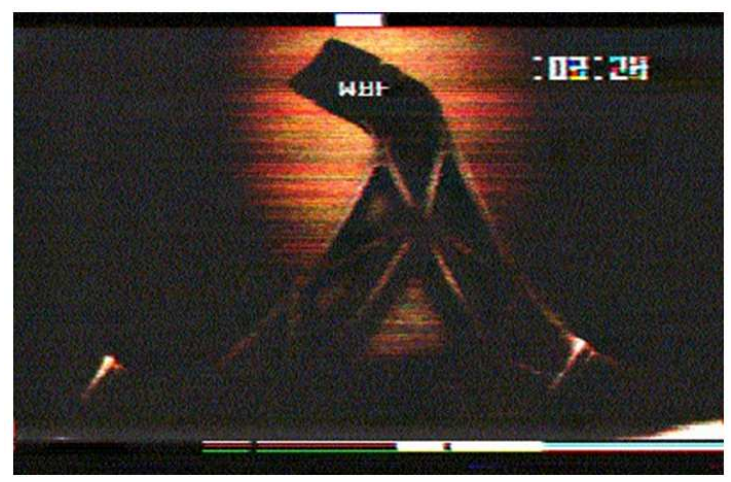

Fig. 9. Undamaged Wishbone thermal image at $3.24 \mathrm{sec}$

\section{DISCUSSION}

Three bands used to analyze the captured thermal images with functions applied to compute image indices. Matrices 1-4 show results for damaged component structure, while matrices 5-8 show index functions applied to non-damaged side of the same component Equation 1-8:

$$
\begin{aligned}
& \operatorname{RMSE}\left(\mathrm{b}_{1}, \mathrm{~b}_{2}, \mathrm{~b}_{3}\right)=\left[\begin{array}{lllll}
0 & 5.4 & 27.2 & 31.3 & 45.1 \\
& 8 & 2 & 8 & 6 \\
& 12.0 & 26.7 & 69.4 & 108.2 \\
0 & 7 & 3 & 5 & 5 \\
& 9.7 & 24.2 & 61.6 & 95.6 \\
0 & 8 & 2 & 8 & 8
\end{array}\right] \\
& \operatorname{RASE}\left(b_{1}, b_{2}, b_{3}\right)=\left[\begin{array}{lllll}
0 & 13.6 & 67.9 & 78.3 & 112.6 \\
& 6 & 2 & 0 & 9 \\
& 12.5 & 27.7 & 72.1 & 112.4 \\
& 4 & 6 & 3 & 3 \\
0 & 12.3 & 30.5 & 77.8 & 120.7 \\
& 4 & 7 & 4 & 5
\end{array}\right] \\
& \text { Deviation }\left(\mathrm{b}_{1}, \mathrm{~b}_{2}, \mathrm{~b}_{3}\right)=\left[\begin{array}{lllll}
0 & 0.0 & -0.8 & 0.2 & 0.3 \\
& 21 & 7 & 0 & 8 \\
& 0.0 & 0.1 & 0.5 & 0.8 \\
0 & 04 & 8 & 7 & 7 \\
& 0.0 & 0.2 & 0.5 & 0.8 \\
0 & 80 & 1 & 7 & 6
\end{array}\right] \\
& \operatorname{Bias}\left(\mathrm{b}_{1}, \mathrm{~b}_{2}, \mathrm{~b}_{3}\right)=\left[\begin{array}{ccccc}
0 & 0.0 & -0.3 & -0.0 & 0.0 \\
& 04 & 70 & 05 & 31 \\
0 & -0.0 & 0 . & 0 . & 0.6 \\
& 04 & 12 & 39 & 04 \\
0 & 0.05 & 0.13 & 0.37 & 0.55
\end{array}\right] \\
& \operatorname{RMSE}\left(\mathrm{b}_{1}, \mathrm{~b}_{2}, \mathrm{~b}_{3}\right)=\left[\begin{array}{lll}
0 & 35.55 & 56.69 \\
0 & 37.76 & 99.80 \\
0 & 25.00 & 86.20
\end{array}\right] \\
& \operatorname{RASE}\left(\mathrm{b}_{1}, \mathrm{~b}_{2}, \mathrm{~b}_{3}\right)=\left[\begin{array}{lll}
0 & 88.71 & 141.44 \\
0 & 39.21 & 103.62 \\
0 & 31.54 & 108.76
\end{array}\right]
\end{aligned}
$$$$
\operatorname{Deviation}\left(\mathrm{b}_{1}, \mathrm{~b}_{2}, \mathrm{~b}_{3}\right)=\left[\begin{array}{ccc}
0 & -0.23 & -0.90 \\
0 & 0.023 & 0.81 \\
0 & 0.20 & 0.83
\end{array}\right]
$$$$
\operatorname{Bias}\left(\mathrm{b}_{1}, \mathrm{~b}_{2}, \mathrm{~b}_{3}\right)=\left[\begin{array}{ccc}
0 & -0.47 & -0.36 \\
0 & 0.035 & 0.54 \\
0 & 0.13 & 0.52
\end{array}\right]
$$ 
While hyperspectral data are very rich in information, processing the hyperspectral data poses several challenges regarding computational requirements, information redundancy removal, relevant information identification and modeling accuracy.

Driven by classification or discrimination accuracy, it is expected that, as the number of hyperspectral bands increases, the accuracy of classification should also increase. Nonetheless, this is not always the case in a developed model. Redundancy in data can cause convergence instability of models. Furthermore, variations due to noise in redundant data propagate through a classification or discrimination model. The same is true of spectral information that has no relation to the feature being classified in the underlying mathematical model. Such information is the same as noise to any statistical model, even if it is unique and accurate. Thus, processing a large number of hyperspectral bands can result in higher classification inaccuracy than processing a subset of relevant bands without redundancy (Zhao et al., 2011; Darvishzadeha et al., 2011; Amro et al., 2011; Aiazzi et al., 2012).

Figure 10 and 11 show RMSE and RASE for the damaged side of the wishbone over three bands. It is noticed that both RMSE and RASE values increases over time as the heat pulse discharges from the component.

Figure 12 represent an RMSE comparison between damaged and undamaged sides of the wishbone.

From the plotted results, it is clear that the heat pulse discharges faster on the undamaged side compared to the damaged one. This is evident when values of RMSE for both are compared over a similar range of time, giving higher RMSE at lower time values for undamaged side compared to the damaged one. This is due to the fact that the roots mean squared error increase as the heat wave disappears, hence increasing differences between the reference image and the subsequent ones. This increase in RMSE is a function of spectral components such as contrast.

Figure 13 show an overall boundary comparison of damaged and undamaged sides of the structure under test. The figure show a clear difference in the values and boundaries of the undamaged side compared to the damaged one as a function of image index analysis and spectral properties.

To enable selection of the best band(s) which gives better information and classification, a principle is adopted to narrow the search space to include only the bands that the various unsupervised methods respectively deem most informative and least redundant. In this study the condition applied is that the best representative band is the one which has no negative values with uniform and smooth transitions.

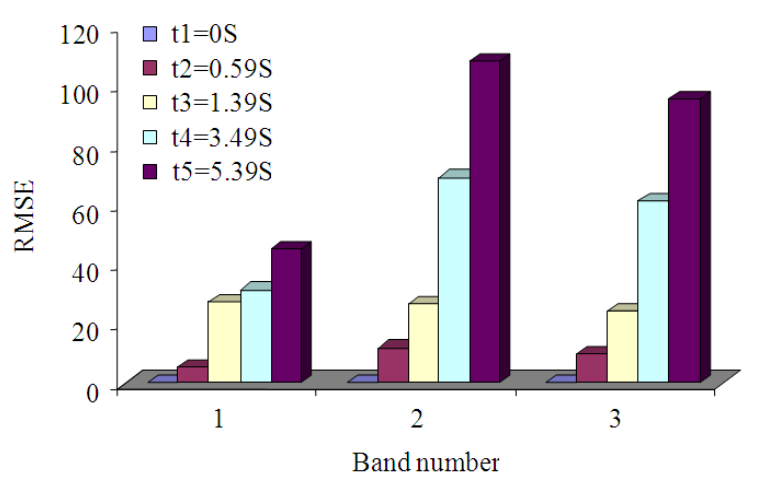

Fig. 10. RMSE of damaged wishbone side

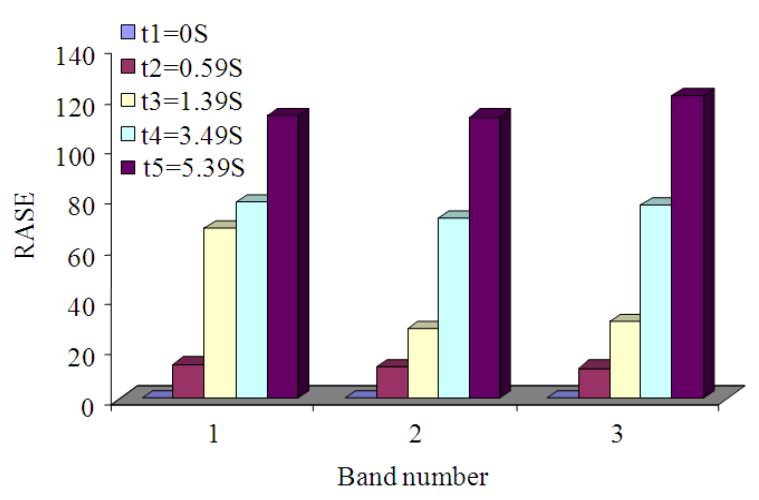

Fig. 11. RASE of damaged wishbone side

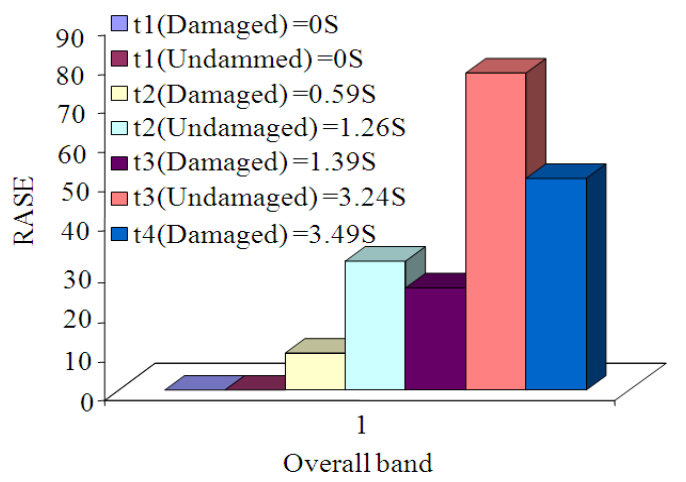

Fig. 12. RMSE for damaged and undamaged sides of wishbone

Hence, a characteristic matrix can now be produced per tested side per selected band. The third band is selected with the following characteristic matrices Equation 9-10:

$\mathrm{C}_{\text {damaged }\left(b_{3}\right)}=\left[\begin{array}{ccccc}0 & 9.78 & 24.22 & 61.68 & 95.68 \\ 0 & 12.34 & 30.57 & 77.84 & 120.75 \\ 0 & 0.08 & 0.21 & 0.57 & 0.86 \\ 0 & 0.05 & 0.13 & 0.37 & 0.55\end{array}\right]$




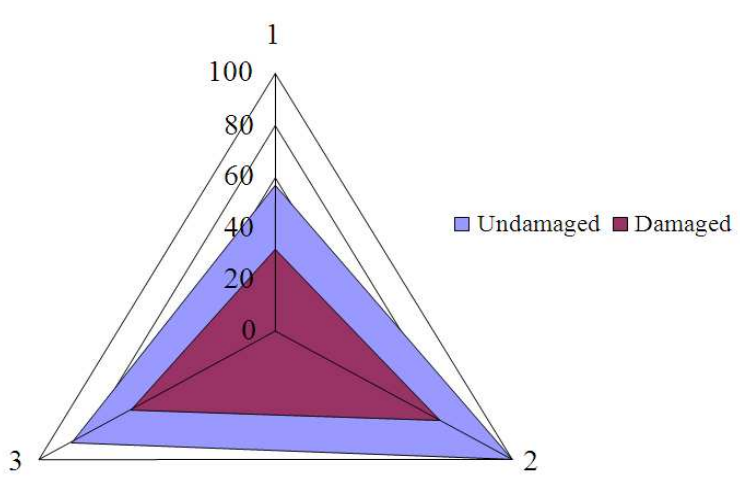

Fig. 13. Boundary comparison of damaged and undamaged wishbone sides

$\mathrm{C}_{\text {Undamaged }\left(\mathrm{b}_{3}\right)}=\left[\begin{array}{ccc}0 & 25.00 & 86.20 \\ 0 & 31.54 & 108.76 \\ 0 & 0.20 & 0.83 \\ 0 & 0.13 & 0.52\end{array}\right]$

From the characteristic matrices, it is realized.

The final value in any computed image index over valid band is the summation of all previous ones; hence, a function can be formed as:

Total Index Value ${ }_{\text {(validband) }}=\sum_{\mathrm{t}_{\mathrm{i}}}^{\mathrm{t}_{\mathrm{i}-1}} \operatorname{Index}(\mathrm{t})$

In this study, the chosen valid band is band $3\left(b_{3}\right)$

The selection of the appropriate band based on the previous conditions that is proved as Equation (11) does not apply for bands $b_{1}$ and $b_{2}$. This is due to the presence of negative values in the Bias and Deviation indices for the damaged part matrices. For the undamaged side, there are negative values in the Bias and Deviation indices of $\mathrm{b} 1$ and unsmooth transition of values in $\mathrm{b} 2$.

As the heat wave of the applied pulse in the undamaged side dissipates so fast, some images could not be captured, but their calculated (lumped index) values that represent image index values over the period of time are described by either Equation 12-15:

$$
\operatorname{Index}_{(\text {calculated })}=\sum_{\operatorname{index}(t>0)}^{\operatorname{Index}(t<1.26)} \operatorname{Index}(t)+\sum_{\operatorname{Index}(t>1.26)}^{\operatorname{Index}(t<3.24)} \operatorname{Index}(t)
$$

Gives:

$\mathrm{C}_{\left.\text {Undamaged ( } b_{3}\right)}=\left[\begin{array}{cccc}0 & 25.00 & 61.20 & 86.20 \\ 0 & 31.54 & 77.22 & 108.76 \\ 0 & 0.20 & 0.63 & 0.83 \\ 0 & 0.13 & 0.39 & 0.52\end{array}\right]$

$$
\operatorname{Index}(\text { calculated })=\sum_{\operatorname{index}(t>3.24)}^{\operatorname{Index}\left(t<t_{\text {final }}\right)} \operatorname{Index}(t)
$$

Gives:

$$
\mathrm{C}_{\text {Undamaged (b } \left.\mathbf{b}_{3}\right)}=\left[\begin{array}{cccc}
0 & 25.00 & 86.20 & 111 \\
0 & 31.54 & 108.76 & 140.3 \\
0 & 0.20 & 0.83 & 1.03 \\
0 & 0.13 & 0.52 & 0.65
\end{array}\right]
$$

In either of the cases above, the undamaged part is distinguished from the damaged one.

Using Equation (14), the damaged part is differentiated from the undamaged one with final column matrices as follows Equation 16-18:

$\mathrm{Cfinal}_{(\text {Damaged })}=\left[\begin{array}{c}95.68 \\ 120.75 \\ 0.86 \\ 0.55\end{array}\right]$

Cfinal $_{\text {(Undamaged) }}=\left[\begin{array}{c}111 \\ 140.3 \\ 1.03 \\ 0.65\end{array}\right]$

Matrices 18 represent Entropy data for the damaged side of the tested structure. The information entropy measure based on the probability density function of reflectance values in a hyperspectral band and the number of distinct reflectance values. The probabilities are estimated by computing a histogram of reflectance values. In general, if the entropy value is high then the amount of information in the data is large but not necessarily useful:

Entropy $\left(b_{1}, b_{2}, b_{3}\right)=\left[\begin{array}{ccccc}6.40 & 6.34 & 7.56 & 6.23 & 6.11 \\ 7.23 & 7.44 & 6.88 & 6.03 & 5.95 \\ 7.13 & 6.66 & 6.43 & 6.18 & 6.10\end{array}\right]$

Figure 14, show how entropy changes over time for energy pulsed wishbone composite structure. From the plot, it is noticed that the only band which has smooth entropy transition that is consistent with the fact that as the spectral properties fade and the difference as in RMSE and Deviation increase, thus the entropy should decrease. This is also consistent with the criteria for band acceptance established earlier. 


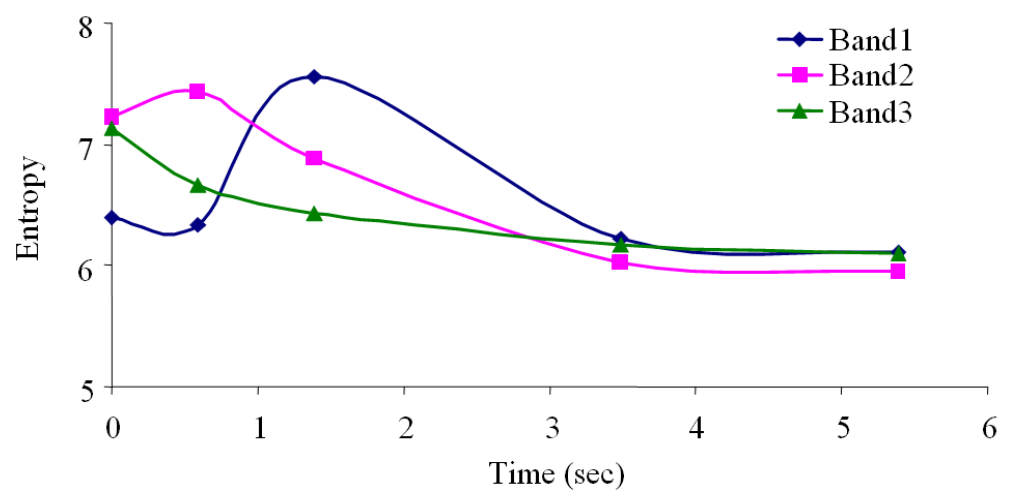

Fig. 14. Entropy as a function of time

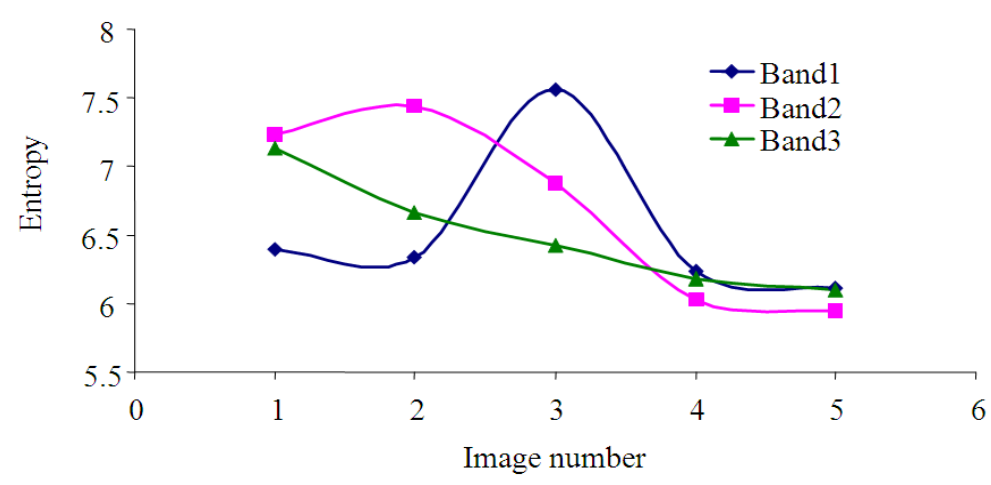

Fig. 15. Entropy as a function of image

Figure 15 shows Entropy change per image, where it is clear that each band gives different information at a different image and different instant of time. However, the only decreasing entropy function as per captured image is represented in band3.

\section{CONCLUSION}

The findings of this study are:

- Ability to detect and discriminate between damaged and undamaged composite structures using Hyperspectral technique

- Using the new approach of time compensation acted as a weight equivalent in Neural Systems, which resulted in accurate classification

- Establishing criteria for band selection in case of PVT and other IR based images

- Ability to predict cumulative values of unregistered past or future images

\section{REFERENCES}

Aiazzi, B., L. Alparone, S. Baronti, C. Lastri and M. Selva1, 2012. Spectral distortion in lossy compression of hyperspectral data. J. Elect. Comput. Eng., 2012: 1-8. DOI: $10.1155 / 2012 / 850637$

Amro, I., J. Mateos, M. Vega, R. Molina and A. Katsaggelos, 2011. A survey of classical methods and new trends in pansharpening of multispectral images. EURASIP J. Adv. Signal Proces., 79: 1-22. DOI: $10.1186 / 1687-6180-2011-79$

Baranowski, P., W. Mazurek, J. Wozniak and U. Majewska, 2012. Detection of early bruises in apples using hyperspectral data and thermal imaging. J. Food Eng., 110: 345-355. DOI: 10.1016/j.jfoodeng.2011.12.038

Cheng, L. and G.Y. Tian, 2012. Comparison of nondestructive testing methods on detection of delaminations in composites. J. Sensors, 2012: 1-7. DOI: 10.1155/2012/408437 
Darvishzadeha, R., C. Atzbergerb, A. Skidmorec and M. Schlerfc, 2011. Mapping grassland leaf area index with airborne hyperspectral imagery: A comparison study of statistical approaches and inversion of radiative transfer models. ISPRS J. Photogrammetry Remote Sens., 66: 894-906. DOI: 10.1016/j.isprsjprs.2011.09.013

Kutin, M., S. Ristic, M. Puharic, M. Vilotijevic and M. Krmar, 2011. Thermographic testing of epoxy-glass composite tensile properties. Contemporary Mater., 2: 88-93. DOI: 10.5767/anurs.cmat.110201.en.088K

Lahiria, B.B., S. Bagavathiappana, P.R. Reshmib, J. Philipa and T. Jayakumara et al., 2012. Quantification of defects in composites and rubber materials using active thermography. Infrared Phys. Technol., 55: 191-199. DOI: 10.1016/j.infrared.2012.01.001

Liu, F., F. Seinstra and A. Plazac, 2011. Parallel hyperspectral image processing on distributed multicluster systems. J. Applied Remote Sens., 5: 114. DOI: $10.1117 / 1.3595292$

Meer, F.D.V.D., H.A.V.D. Werff, F.A.V. Ruitenbeek, C.A. Hecker and W.H. Bakker et al., 2012. Multiand hyperspectral geologic remote sensing: A review. Int. J. Applied Earth Observation Geoinform., $\quad 14$ : $112-128 . \quad$ DOI: 10.1016/j.jag.2011.08.002
Myriounis, D.P., E.Z. Kordatos, S.T. Hasan and T.E Matikas, 2011. crack-tip stress field and fatigue crack growth monitoring using infrared lock-in thermography in a359/SICP composites. Strain, 47: e619-e627. DOI: $\quad 10.1111 / \mathrm{j} .1475-$ 1305.2009.00665.x

Naderi, M., A. Kahirdeh and M.M. Khonsari, 2012. Dissipated thermal energy and damage evolution of Glass/Epoxy using infrared thermography and acoustic emission. Comp. Part B: Eng., 43: 16131620. DOI: $10.1016 /$ j.compositesb.2011.08.002

Picon, A., O. Ghita, A. Bereciartua, J. Echazarra and P. Whelan et al., 2012. Real-time hyperspectral processing for automatic nonferrous material sorting. J. Elect. Imag., 21: 13-18. DOI: 10.1117/1.JEI.21.1.013018

Wu, C.Q., W.P. Wang, Q.G. Yuan, Y.J. Li and W. Zhang et al., 2011. Infrared thermography nondestructive testing of composite materials. Adv. Mater. Res., 291-294: 1307-1310: DOI: 10.4028/www.scientific.net/AMR.291-294.1307

Yanga, C., J. Goolsbya, J. Everitta and Q. Dub, 2012. Applying six classifiers to airborne hyperspectral imagery for detecting giant reed. Geocarto Int., 1-2: 1-12. DOI: $10.1080 / 10106049.2011 .643321$

Zhao, Y., J. Yang, Q. Zhang, L. Song and Y. Cheng et al., 2011. Hyperspectral imagery super-resolution by sparse representation and spectral regularization. EURASIP J. Adv. Signal Process., 87: 1-10. DOI: 10.1186/1687-6180-2011-87 